\title{
Exercise prescription and the doctor's duty of non-maleficence
}

\author{
Jonathan Pugh, ${ }^{1}$ Christopher Pugh, ${ }^{2,3}$ Julian Savulescu ${ }^{1}$
}

An abundance of data unequivocally demonstrates that exercise can be an effective tool in the fight against obesity and its associated comorbidities. ${ }^{1}$ Indeed, physical activity can be more effective than widely used pharmaceutical interventions. While metformin reduces the incidence of diabetes by $31 \%$ (as compared with a placebo) in both men and women across different racial and ethnic groups, lifestyle intervention (including exercise) reduces the incidence by $58 \%$. $^{2}$

In this context, it is notable that a group of prominent medics and exercise scientists recently sent a well-publicised letter to the General Medical Council (GMC) and Medical Schools Council calling for the introduction of evidencebased lifestyle education into all medical curricula. ${ }^{3}$ The letter warns that there is a lack of understanding of the impact that exercise and nutrition can have on physical health among doctors. In the absence of an educational overhaul, the signatories warn that the government is likely to fail to reach its goal of preventing tens of thousands of premature deaths from heart disease and cancer by 2020 .

While we agree with the need to address this apparent lack of understanding, the ethical justification of doing so is not limited to this broadly beneficence-based justification. There is also a justification grounded in the duty of non-maleficence, that is, the duty to avoid unreasonable harm to patients.

Despite the well-established long-term beneficial effects of exercise, the risk of an acute cardiovascular event may be transiently elevated during and just after vigorous physical exertion for susceptible individuals. This is the so-called 'paradox of exercise'. ${ }^{4}$ This paradox does not mean doctors should refrain from prescribing exercise; the long-term beneficial effects of exercise far outweigh the acute risks. ${ }^{5}$

\footnotetext{
${ }^{1}$ Uehiro Centre for Practical Ethics, University of Oxford, Oxford, UK

${ }^{2}$ School of Sport, Cardiff Metropolitan University, Cardiff, UK

${ }^{3}$ Cardiff Centre for Exercise and Health, Cardiff Metropolitan University, Cardiff, UK
}

Correspondence to Professor Julian Savulescu, Uehiro Centre for Practical Ethics, University of Oxford, Suite 8, Littlegate House, 16/ 17 St Ebbe's St, Oxford OX1 2JD, UK ; julian.savulescu@philosophy.ox.ac.uk

\section{Box 1 Reasonable risk}

In determining whether the risks of participation in exercise are reasonable, the following factors are relevant: ${ }^{78}$

1. Is there a known risk to participants prior to commencing exercise and what is its magnitude, based on evidence available at the time? Are there relevant evidence-based professional guidelines (eg. American College of Sports Medicine guidelines) to categorise the risk to this participant?

2. Should any further research(eg, systematic overview or computer modelling) be performed prior to the exercise to better estimate the risk to particular participants?

3. Could the risk be reduced in any other way? Is it as small as possible?

4. Are the potential benefits (in terms of health and global well-being) of exercise worth the risks?

Indeed, low levels of physical activity are a significant contributing factor to whether a particular individual is susceptible to the elevated risk of sudden death during exercise. This risk is dramatically lower in regular exercisers and dramatically higher in habitually sedentary individuals who undertake a sudden bout of unaccustomed vigorous exercise. ${ }^{6}$ However, doctors have a moral reason, grounded in the duty of non-maleficence (ie, the duty to not harm patients) to refrain from (1) preventing patients from undergoing beneficial treatment without good reason, (2) exposing patients to unreasonable risks (we define reasonable risk in box 1), and (3) reducing the therapeutic effect of an effective medical intervention. This requires an understanding of the physical impact of exercise.

Although doctors have the necessary expertise to identify conventional medical risk factors for cardiac events during exercise, a lack of understanding of the physical impact of exercise might lead doctors to overemphasise these risk factors. Under the recently updated American College of Sports Medicine (ACSM) exercise preparticipation health screening process, medical clearance is only recommended for exercise if the individual has already been identified as at risk of an acute cardiovascular event based on pre-exercise risk factor screening. ${ }^{9}$ According to these guidelines, whether or not an individual should receive medical clearance prior to exercise depends on the individual's history of physical activity and the individual's desired exercise intensity (as well as the presence of signs or symptoms of known cardiovascular, metabolic or renal disease). ${ }^{9}$ A lack of understanding of the importance of these other risk factors might lead doctors to either underprescribe exercise for patients with known cardiovascular, metabolic or renal disease, or dissuade them from exercise by insisting on tests prior to exercise, tests that the scientific community has agreed are unnecessary. ${ }^{9}$

Second, we cannot ignore the possibility that this lack of understanding might lead doctors to unnecessarily prescribe exercise interventions with a higher degree of relative risk. The National Health Service guidelines for physical activity in adults recommend at least 150 minutes of moderate aerobic activity for adults per week. This recommendation is well supported and well-established. However, these guidelines also advocate the heuristic that ' 1 minute of vigorous activity provides the same health benefits as 2 minutes of moderate activity'. ${ }^{10}$ Yet, for habitually inactive patients, the relevant trade-off here is not just between exercising for 10 minutes versus 20 minutes to achieve the same health outcome-it is also a choice between interventions that expose these individuals to significantly different degrees of relative risk of suffering a cardiac event in trying to achieve the same health outcome. Crucially though, this higher relative risk for inactive individuals can easily be avoided by a gradual progression towards vigorous exercise. ${ }^{11}$ In view of the definition provided in box 1 , in particular factors 1 and 3, this may constitute exposing patients to unreasonable risk. Recognising this is all the more important following the Montgomery ruling governing claims of medical negligence: doctors need to be increasingly aware of even small risks of medical interventions, the potential need to disclose these risks, and to offer alternatives when possible.

Finally, a lack of understanding of the interactions between pharmaceuticals and exercise could lead doctors to prescribe treatments that are not only less effective than exercise, but which might also serve to blunt its therapeutic effect. A recent study suggests that metformin may attenuate the effects of exercise on certain 
cardiovascular risk factors and the severity of metabolic syndrome in patients with impaired glucose tolerance. ${ }^{12}$ Even more strikingly in this context, some statins may attenuate the exercise-mediated increases in cardiorespiratory fitness in obese or overweight patients. ${ }^{13}$

Further large-scale studies are required to confirm the relationship between these medications and the exercise intervention outcomes. However, these studies raise the possibility that prescribing these drugs rather than exercise for certain patients might not simply mean foregoing the benefit of a more effective treatment; if the patient has already adopted lifestyle changes to combat their disease, these drugs might reduce the considerable therapeutic effect of these lifestyle changes.

Exercise prescription can be a powerful weapon in the fight against obesity and its associated comorbidities. However, in order to maximise the therapeutic benefits of exercise and to avoid the unnecessary harm outlined above, it must be implemented by professionals with an adequate understanding of the impact that exercise can have on physical health. If doctors are expected to be at the vanguard of exercise prescription, and assuming they can be said to apparently lack the aforementioned understanding, our arguments lend support to the conclusions reached in the recent letter sent to the GMC. However, we also note that there will be significant costs associated with overhauling medical education in the way that the signatories of the letter advocate, and that doctors are already highly overburdened in the
UK. As such, our arguments may also be taken to highlight the crucial importance of considering alternative ways in which it may be possible to bring existing expertise regarding the impact of exercise on physical health to bear at the coalface in medicine. Professionals with training in exercise prescription and under the governance of the Faculty of Sport and Exercise Medicine UK would be well placed to fill this lacuna, in light of their grasp of the relevant evidence and guidelines, and their ability to assess reasonable risk in this context.

Contributors All authors contributed equally to the manuscript.

Funding Julian Savulescu's contribution to this manuscript was funded by the Wellcome Trust, grant number WT104848/Z/14/Z.

\section{Competing interests None declared.}

Provenance and peer review Not commissioned; externally peer reviewed.

(C) Article author(s) (or their employer(s) unless otherwise stated in the text of the article) 2017. All rights reserved. No commercial use is permitted unless otherwise expressly granted.

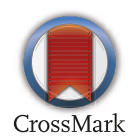

To cite Pugh J, Pugh C, Savulescu J. Br J Sports Med 2017;51:1555-1556.

Accepted 10 March 2017

Published Online First 29 March 2017

Br J Sports Med 2017:51:1555-1556.

doi:10.1136/bjsports-2016-097388

\section{REFERENCES}

1 Warburton DER, Nicol CW, Bredin SSD. Health benefits of physical activity: the evidence. Can Med Assoc J 2006;174:801-9.
2 Knowler WC, Barrett-Connor E, Fowler SE, et al. Diabetes Prevention Program Research Group. Reduction in the incidence of type 2 diabetes with lifestyle intervention or metformin. N Eng/ J Med 2002;346:393-403.

3 Campbell D. Doctors "know too little about nutrition and exercise." The Guardian 19 October 2016. https:// www.theguardian.com/society/2016/oct/19/doctorsknow-too-little-about-effects-of-nutrition-and-exercise (accessed 4 Nov 2016).

4 Maron BJ. The paradox of exercise. N Engl J Med 2000;343:1409-11.

5 Thompson PD, Franklin BA, Balady GJ, et al. American Heart Association Council on Nutrition, Physical Activity, and Metabolism American Heart Association Council on Clinical Cardiology American College of Sports Medicine. Exercise and acute cardiovascular events placing the risks into perspective: a scientific statement from the American Heart Association Council on Nutrition, Physical Activity, and Metabolism and the Council on Clinical Cardiology. Circulation 2007;115:2358-68.

6 Mittleman MA, Mostofsky E, Physical ME. Physical, psychological and chemical triggers of acute cardiovascular events: preventive strategies. Circulation 2011;124:346-54.

7 Savulescu J, Hope T Ethics of research In: Skorupski $\mathrm{J}$ ed. The Routledge companion to ethics. Abingdon: Routledge, 2010:781-95.

8 Savulescu J. Commentary: safety of participants in non-therapeutic research must be ensured. BMJ 1998;316:891-4.

9 Riebe D, Franklin BA, Thompson PD, et al. Updating ACSM's recommendations for exercise preparticipation health screening. Med Sci Sports Exerc 2015;47:2473-9.

10 NHS Choices. Physical activity guidelines for adultsLive well. 2016 http://www.nhs.uk/Livewell/fitness/ Pages/physical-activity-guidelines-for-adults.aspx.

11 Goodman J, Thomas S, Burr JF. Cardiovascular risks of physical activity in apparently healthy individuals. Can Fam Physician 2013;59:46-9.

12 Malin SK, Nightingale J, Choi SE, et al. Metformin modifies the exercise training effects on risk factors for cardiovascular disease in impaired glucose tolerant adults. Obesity 2013;21:93-100.

13 Mikus CR, Boyle LJ, Borengasser SJ, et al. Simvastatin impairs exercise training adaptations. J Am Coll Cardiol 2013;62:709-14. 